10. Webb EM, Green GE, Scoutt LM. Adnexal mass with pelvic pain. Radiol Clin North Am 2004;42:329-48.

11. Cappell MS, Friedel D. Abdominal pain during pregnancy. Gastroenterol Clin North Am 2003;32:1-58.

12. Gorkemli H. Adnexal torsion after gonadotrophin ovulation induction for IVF or ICSI and its conservative treatment. Arch Gynecol Obstet 2002;267:4-6.

13. Burnett LS. Gynecological causes of the acute abdomen. Surg Clin North Am 1988;68:385-98.

14. Morgan A. Genitourinary emergencies: adnexal mass evaluation in the emergency department. Emerg Med Clin North Am 2001;19:799-816.
15. Pena JE, Ulfberg D, Cooney N, et al. Usefulness of Doppler sopnography in the diagnosis of ovarian torsion. Fertil Steril 2000;73:1047-50.

16. Cohen SB. Laparoscopic detorsion allows sparing of the twisted ischemic adnexa. J Am Assoc Gynecol Laparosc 1999;6:139-43.

17. Zweizig S, Perron J, Grubb D, et al. Conservative management of adnexal torsion. Am J Obstet Gynecol 1993;168:1791-5.

Correspondence to: Dr. Kirk Magee, Department of Emergency Medicine, 3021 Halifax Infirmary, QE II Health Sciences Centre, 1796 Summer St., Halifax NS B3H 3A7

\title{
Calendar • Calendrier
}

\section{CAEP 2006 Roadshows}

\section{Register online for CAEP events. Additional sites may be arranged upon request. www.caep.ca}

\begin{abstract}
AIME: Airway Interventions \& Management Education
Toronto, Ont. [prior to North York EM Update] Halifax, NS [prior to ICEM 2006]

May $3 \& 4$

June $2 \& 3$
\end{abstract}

\section{EDTU - ED Targeted Ultrasound}

Antigonish, NS

Halifax, NS [prior to ICEM 2006]

Muskoka, Ont.

April 22

June $2 \& 3$

July 8 \& 9

\section{ED STAT! - ED Strategies for Teaching Any Time}

\section{Calgary, Alta.}

Thunder Bay, Ont.

Prince George, BC

San Francisco, Calif. [at SAEM]

Halifax, NS [prior to ICEM 2006]

\section{Kids CAEP-ERs: a Day in the ED}

Cambridge, Ont. [prior to Tri-City EM Conference] Summerside, PEI

Regina, Sask.

Mar. $20 \& 21$

Mar. 25

Apr. 22

May 20

June 3

Roadshows info: Janice Maclsaac, CME Coordinator; 800 463-1158 x20, fax 613 523-0190, cme-

registration@caep.ca

\section{ICEM 2006}

\section{1th International Conference on EM}

Halifax, NS

June 3-7

Contact: Compart Event Management; 902 454-4714, fax 902 454-4930, icem2006@compartevents.com; www.icem2006.com

\section{Other CME Programs}

\section{EM \& Acute Care Series}

Costa Rica

Mar. 9-13

Orlando, Fla.

Apr. 27-30

[discounted $\$ 100$, with pay't to EMA by credit card in US funds] Contact: Emergency Medical Abstracts; 800 458-4779.

Go to www.ccme.org for complete list of courses.

\section{Tri-City ER Conference}

Holiday Inn, Cambridge, Ont.

Mar. 29

Contact: Guelph General Hospital; 519 837-6440 ×2588,

kcrawford@gghorg.ca

\section{Herb Parkin Day in EM 2006}

Coquitlam, BC

Mar. 31

Contact: Medical Education Department, Royal Columbian Hospital; 604 520-4211, Barb.Geddes@fraserhealth.ca

\section{Paediatric Update 2006}

Hospital for Sick Children, Toronto, Ont. May 1-6 Contact: CME; brenda.rau@sickkids.ca, www.sickkids.ca/Health careProfessionals/default.asp

\section{North York EM Update}

Royal York Hotel, Toronto, Ont.

May 4-6

Contact: North York General Hospital Conference Services;

Mar. 28

May 10

May 26

416 635-2522, cme@nygh.on.ca, www.nygh.on.ca

\section{Society for Academic Emergency Medicine (SAEM)} Annual General Meeting

San Francisco, Calif. May 18-21

Contact: 517 485-5484, fax 517 485-0801, saem@saem.org

\section{Child Maltreatment Conference}

Hospital for Sick Children, Toronto, Ont.

June $8 \& 9$

Contact: CME; brenda.rau@sickkids.ca, www.sickkids.ca/Health careProfessionals/default.asp

\section{Congrès scientifique de MU}

Centre des congrès de Québec

Oct. 24-27

Contactez : L'Association des médecins d'urgence du Québec; 418 658-7679, amuq@amuq.gc.ca, www.amuq.gc.ca

Don't forget! CAEP 2006 is ICEM 2006. For more information visit www.caep.ca and click on ICEM2006. 